\title{
QUALIDADE MICROBIOLÓGICA E FÍSICO-QUÍMICA DE SUCOS DE LARANJA COMERCIALIZADOS NAS VIAS PÚBLICAS DE PORTO ALEGRE/RS
}

\author{
Carla K. RUSCHEL ${ }^{2}$, Heloisa H. CARVALHO², \\ Roberval B. de SOUZA², Eduardo C. TONDO', *
}

\begin{abstract}
RESUMO
O comércio de sucos de laranja não pasteurizados vem aumentando nos últimos anos na cidade de Porto Alegre/RS. Com o intuito de avaliar a qualidade desses produtos, análises físicoquímicas e microbiológicas foram realizadas em 52 amostras de sucos de laranja comercializados nas vias públicas de Porto Alegre. As análises microbiológicas demonstraram 44,23\% ( $n=23)$ das amostras, em desacordo com os padrões estabelecidos pela Legislação Federal vigente, sendo os bolores e leveduras encontrados em níveis inapropriados em todas as amostras reprovadas. Coliformes fecais foram encontrados acima das quantidades permitidas em 5,7\% ( $n=3)$ das amostras e Salmonella cholerasuis foi identificada em uma das amostras analisadas. As análises físico-químicas apresentaram $89,47 \%$ das amostras em desacordo com pelo menos um dos parâmetros analisados. Com base nestes resultados, sugerem-se maiores cuidados nas boas práticas de fabricação (BPF), principalmente na higiene pessoal de manipuladores e sanificação de máquinas extratoras.
\end{abstract}

Palavras-chave: suco de laranja; análises microbiológicas; análises físico-químicas.

\section{SUMMARY}

MICROBIOLOGICAL AND PHYSICAL-CHEMICAL QUALITY OF ORANGE JUICES COMMERCIALISED IN PUBLIC STREETS OF PORTO ALEGRE CITY. The commercialisation of nonpasteurized orange juice has been increased in recent years in Porto Alegre city, in South Brazil. The purpose of this research was to evaluate the quality of this product. Physical-chemical and microbiological analyses were performed in 52 samples of orange juice purchased in the streets of Porto Alegre city. The microbiological analysis demonstrated that $44,23 \%(n=23)$ of the samples did not agree with Federal Regulations. All the reproved samples presented inappropriate levels of molds and yeasts. Fecal coliforms were found in 5,7\% ( $n=3)$ of the samples, while Salmonella Cholerasuis was isolated from one sample. The physical-chemical analysis demonstrated that $89,47 \%$ of the samples were in discordance with parameters established by Federal Regulations. Based on the results, good manufacturing practices (GMP), specially on personnel hygienic habits, and equipment sanitization are necessary.

Keywords: orange juice; microbiological analysis; physical-chemical analysis

\footnotetext{
${ }_{1}^{1}$ Recebido para publicação em 31/10/00. Aceito para publicação em 02/03/01.

${ }^{2}$ Instituto de Ciência e Tecnologia de Alimentos - ICTA/UFRGS, Av. Bento Gonçalves, 9500. Prédio 43212, Caixa Postal 15090, CEP 91505-970, Campus do Vale, Bairro Agronomia, Porto Alegre /RS, BRASIL

* A quem a correspondência deve ser enviada.
}

\section{1 - INTRODUÇÃO}

Em conseqüência das adversidades econômicas brasileiras, cresce cada vez mais a procura por alternativas viáveis de subsistência. $\mathrm{O}$ aumento do comércio paralelo, assim como o grande número de micro e pequenas empresas tem demonstrado a agilidade no aproveitamento de oportunidades de comércio. Contudo, esse desenvolvimento comercial, principalmente no ramo de alimentação, deve ser acompanhado pela garantia de qualidade a fim de assegurar bases sólidas e legais aos produtos alimentícios.

O baixo custo de produção, assim como a aceitabilidade dos sucos de laranja in natura têm proporcionado o desenvolvimento do mercado desse produto em diferentes capitais brasileiras [1, 7]. Além disso, o mercado formal de sucos de laranja cresce a nível mundial, conquistando clientela exigente mesmo competindo com produtos de renome [9]. Um dos principais fatores para tal desenvolvimento é o investimento em qualidade. Nesse tipo de produto, as características físico-químicas e o conteúdo microbiológico são fundamentais, uma vez que deles dependerão o atendimento aos padrões de identidade e qualidade e o "shelflife", possibilitando ou não a sua comercialização. Como fatores que influenciam a condição microbiológica dos sucos de laranja, pode-se destacar as condições higiênico-sanitárias das extratoras, além do treinamento de pessoal que nelas trabalham. Boa parte das pequenas indústrias produtoras de suco de laranja em Porto Alegre estão equipadas com espremedores automáticos, aos quais são adicionadas diretamente as laranjas depois de prévia lavagem das cascas. Freqüentemente, esses equipamentos não são corretamente higienizados, podendo tornarem-se fontes de contaminação principalmente por bolores e leveduras. Tanto os proprietários quanto os manipuladores dessas indústrias muitas vezes, não têm acesso à informação ou treinamento para produção de alimentos, ocasionando adulterações nos padrões físico-químicos e contaminações microbianas variadas como os coliformes fecais, Salmonella ou outros microrganismos não investigados pelas fiscalizações. Além disso, essas pequenas indústrias distribuem os sucos de laranja nas vias públicas em carrinhos de fibra de vidro refrigerados com gelo em barra ou em cubos, os quais comprometem a qualidade de refrigeração principalmente ao final dos dias quentes.

Outro fator importante a ser ressaltado é a falta de preparo dos vendedores dos sucos de laranja. Não raro nas vias públicas de Porto Alegre, pode-se acompa- 
nhar a falta de higiene dos mesmos ou hábitos que podem comprometer a qualidade do produto como deixá-lo fora de refrigeração, ou manter os canudos em lugares inapropriados.

Em vista do desenvolvimento do comércio de sucos de laranja não pasteurizados e engarrafados, esse trabalho objetivou analisar as características higiênico-sanitárias e físico-químicas dos sucos de laranja comercializados em vias públicas de Porto Alegre/RS.

\section{2 - MATERIAIS E MÉTODOS}

Durante o período de março de 1996 a janeiro de 1998, foram analisadas quanto ao seu conteúdo microbiológico, 52 amostras de suco de laranja não pasteurizado engarrafado. Destas, 38 amostras foram também analisadas quanto às suas características físico-químicas. Os sucos de laranja foram entregues aos laboratórios de análises físico-químicas e microbiológicas do Instituto de Ciência e Tecnologia de Alimentos/UFRGS, para fins de registro frente ao Ministério da Agricultura (38 amostras com análises físico-químicas e microbiológicas) ou coletadas nas vias públicas de Porto Alegre (14 amostras analisadas microbiologicamente) sempre dentro do prazo de validade do produto. Os resultados das análises microbiológicas foram comparados aos padrões estabelecidos pela Portaria 451 de 19/09/97 do Ministério da Saúde [4] e as análises fisico-químicas obedeceram os limites da portaria DINAL № 1 de 28/01/87 do Ministério da Agricultura e do Abastecimento [3].

As análises microbiológicas consistiram na investigação da presença de Salmonella spp., número mais provável de coliformes fecais e contagem de bolores e leveduras, segundo metodologias descritas por American Public Health Association [16]. A identificação sorológica de Salmonella foi realizada no Laboratório de Microbiologia da Faculdade de Veterinária da UFRGS.

Análises fisico-químicas foram realizadas investigando os seguintes parâmetros: densidade a $20^{\circ} \mathrm{C}$, sólidos solúveis em 'Brix, açúcares totais, acidez em quantidade de ácido cítrico, relação ${ }^{\circ}$ Brix/acidez, ácido ascórbico, e sólidos em suspensão conforme metodologias descritas pelo Instituto Adolfo Lutz [8].

\section{3 - RESULTADOS E DISCUSSÃO}

A Tabela 1 apresenta os resultados das análises microbiológicas

Como pode ser visualizado na Tabela 1, das 52 amostras analisadas microbiologicamente, 44,23\% (23) apresentaram contagens de bolores e leveduras acima dos padrões estabelecidos pela portaria 451/97 - MS. Os valores variaram entre $1 \times 10^{4}$ até $8,2 \times 10^{5} \mathrm{UFC} / \mathrm{mL}$ com média aritmética de $1,27 \times 10^{5} \mathrm{UFC} / \mathrm{mL}$, sugerindo problemas relacionados com a limpeza das cascas das laranjas antes de serem espremidas [1] ou higienização inadequada do maquinário das extratoras [7]. A contaminação por esses tipos de microrganismos não envolve demasiados riscos à saúde humana, uma vez que sucos de laranja não são meios ideais para a pro- dução de micotoxinas [17], contudo SCHEIDEGGER et al [15] sugerem cuidado, haja visto que esses produtos podem ser fontes de severas infecções por Candida albicans em pessoas imuno-deprimidas. Além disso, diversos autores têm demonstrado crescimento de diferentes tipos de fungos, mesmo sob refrigeração, provocando acúmulo de biomassa, redução da quantidade de açúcares $[2,5,17]$ e, conseqüentemente, redução da vida-de-prateleira [12] de sucos de laranja.

TABELA 1. Condição microbiológica dos sucos de laranja não pasteurizados comercializados na cidade de Porto Alegre/RS.

\begin{tabular}{|c|c|c|}
\hline Microrganismos & $\mathrm{N}^{\circ}$ de amostras & $\%$ \\
\hline \multicolumn{3}{|l|}{ Bolores e leveduras (UFC/mL) } \\
\hline amostras adequadas $\left(<10^{4} \mathrm{UFC} / \mathrm{mL}\right)$ & 29 & 55,77 \\
\hline amostras inadequadas (>10 $\mathrm{UFC} / \mathrm{mL})$ & 23 & 44,23 \\
\hline \multicolumn{3}{|l|}{ Coliformes fecais } \\
\hline Amostras adequadas $(<10 \mathrm{NMP} / \mathrm{mL})$ & 49 & 94,23 \\
\hline amostras inadequadas (> $10 \mathrm{NMP} / \mathrm{mL}$ ) & 03 & 5,76 \\
\hline \multicolumn{3}{|l|}{ Salmonella spp. } \\
\hline amostras adequadas (ausência em $25 \mathrm{~mL}$ ) & 51 & 98,07 \\
\hline amostras inadequadas (presença em $25 \mathrm{~mL}$ ) & 01 & 1,92 \\
\hline
\end{tabular}

A contaminação por coliformes fecais acima dos padrões permitidos foi verificada em 5,76\% (3) das 52 amostras analisadas. Os níveis de contaminação verificados foram 11, 11 e $1100 \mathrm{NMP} / \mathrm{mL}$ nas 3 amostras citadas, sugerindo falta de boas práticas de fabricação nas extratoras analisadas. Resultados semelhantes foram encontrados por IHA et al [7] onde $11,4 \%$ de 114 amostras de suco de laranja coletados nas ruas de Ribeirão Preto e Araraquara/SP apresentaram coliformes fecais acima dos padrões estabelecidos por lei. ARRUDA, CARDONHA [1], analisando sucos de laranja comercializados na cidade de Natal/RN, encontraram contaminação inadequada por esses microrganismos em $70 \%$ das 20 amostras coletadas. FURLANETTO, NASCIMENTO [11], em 1981, encontraram 6\% de contaminação por coliformes fecais das 100 amostras de suco de laranja investigadas. A presença de coliformes fecais sugere a possibilidade de demais patógenos em um produto alimentício. A resistência a pHs ácidos apresentada por microrganismos como a E. coli O157:H7 já foi sugerida, como uma das causas de surtos envolvendo produtos ácidos como a cidra de maçã, levantando dúvidas sobre a segurança de sucos de frutas não pasteurizados [10]. PAO, DAVIS [13] demonstraram que a imersão de frutas em água quente $\left(80^{\circ} \mathrm{C}\right)$ por 1 minuto é suficiente para reduzir significativamente o número de $E$. coli na superfície de frutas antes do processamento. Esses autores sugerem ser essa uma medida preventiva para problemas de saúde e apodrecimento precoce dos sucos de fruta.

A presença de Salmonella cholerasuis foi verificada em uma das 52 amostras investigadas pelo presente estudo, sugerindo potencial risco à saúde de consumi- 
dores e falta de processamentos adequados durante a fabricação dos sucos de laranja. PARISH [14] relatou a ocorrência de seis surtos envolvendo o consumo de sucos de laranja não pasteurizados, sendo que pelo menos três deles foram causados por Salmonella. D'AOUST [6] salienta que a presença de Salmonella ácido-tolerante já foi verificada em diversos tipos de produtos, aumentando a probabilidade de problemas de saúde pública, uma vez que essas linhagens podem não ser inativadas pela ação antimicrobiana dos ácidos estomacais e sobreviverem dentro de polimorfos nucleares.

A Tabela 2 apresenta os resultados das análises físico-químicas dos sucos de laranja amostrados.

TABELA 2. Características físico-químicas de sucos de laranja comercializados na cidade de Porto Alegre/RS

\begin{tabular}{lclc}
\hline \multicolumn{1}{c}{ Análise } & $\mathrm{n}$ & Média $\pm \mathrm{DP}$ & Amostras inadequadas (\%) \\
\hline Densidade a $20{ }^{\circ} \mathrm{C}$ & 38 & $1,03 \pm 0,012$ & 57,89 \\
oBrix a 20 ${ }^{\circ} \mathrm{C}$ & 38 & $9,74 \pm 3,04$ & 50 \\
Açúcares totais (\%) & 38 & $8,26 \pm 2,95$ & 0 \\
Acidez (\% Ác.cítrico) & 38 & $0,97 \pm 0,63$ & 0 \\
oBrix/Ácidez & 38 & $14,42 \pm 10,00$ & 36,84 \\
Ácido ascórbico (mg/\%) & 38 & $32,11 \pm 16,63$ & 44,73 \\
Sólidos em suspensão (\%) & 38 & $6,62 \pm 1,8$ & 50
\end{tabular}

DP: desvio-padrão

O suco de laranja é um produto natural e não pode ser adicionado de quaisquer aditivos para correção de parâmetros físico-químicos [7]. Os valores apresentados na Tabela 2 demonstram que 57,89\% (22) das 38 amostras analisadas estavam com a densidade a $20^{\circ} \mathrm{C}$ fora dos padrões especificados, assim como 50\% (19) das amostras demonstraram anormalidades nos sólidos solúveis em ${ }^{\circ}$ Brix e nos sólidos em suspensão. A análise dos sucos coletados demostrou que 36,84\% (14) e $44,73 \%$ (17) dos mesmos apresentaram padrões

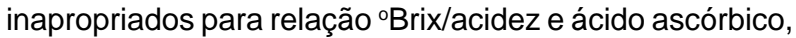
respectivamente. Apenas os testes de açúcares totais e acidez (ácido cítrico) apresentaram valores apropriados para todas as amostras analisadas. As análises físico-químicas demonstraram muitas amostras de suco com inconformidades. Apenas 4 amostras (10,58\%) apresentaram-se de acordo com os padrões legais vigentes. A grande variação nesses parâmetros pode ser atribuída principalmente à variabilidade das características dos frutos utilizados ou à adição de água aos sucos de laranja analisados. Análises físico-química de sucos de laranja engarrafado (produto final) e do suco das laranjas (matéria-prima) extraídos no próprio laboratório foram realizadas em paralelo (resultados não apresentados), demonstrando que a qualidade e, inclusive, conformidade legal dos sucos dependem, significativamente, das características do fruto utilizado. Mesmo assim, a possibilidade de adulteração freqüente não pode ser descartada.

\section{4 - CONCLUSÕES}

Em vista dos resultados apresentados, maior controle sanitário de pessoal e de equipamentos devem ser empregados durante a fabricação de sucos de laranja engarrafados não pasteurizados. Além disso, atenção deve ser direcionada no estabelecimento de novos padrões de análise físico-química, a fim de diferenciar adulterações das variações das características naturais dos frutos utilizados nos sucos de laranja.

\section{5 - REFERÊNCIAS BIBLIOGRÁFICAS}

[1] ARRUdA, W. R.; CARDONHA, A. M. S. Avaliação microbiológica de sucos de laranja "in natura" comercializados na cidade de Natal-RN. XVI Congresso Brasileiro de Ciência e Tecnologia de Alimentos - SBCTA, Rio de Janeiro, 1998.

[2] BEUCHAT, L. R. Spoilage of acid products by heat-resistant molds. Dairy, Food and Environmental Sanitation, v.18, n 9, p.588-593, 1998.

[3] BRASIL. Ministério da Agricultura e do Abastecimento. Serviço de Inspeção Vegetal- DFA/RS. Portaria DINAL número 01 de 28 de janeiro de 1987. Brasília, Min. Agr. Abast., 1987.

[4] BRASIL. Portaria no 451, de 19 de setembro de 1997. Regulamento técnico princípios gerais para o estabelecimento de critérios e padrões microbiológicos para alimentos. Diário Oficial da República Federativa do Brasil, Brasília, n. 182, p. 21005-21011, 22 set. 1997. Seção 1.

[5] CANCALON, P. F., PARISH, M. E. Changes in the chemical composition of orange juice during growth of Saccharomyces cerevisiae and Gluconobacter oxydans. Food Microbiology, v.12, p.117-124, 1995.

[6] D'AOUST, J. Y. Salmonella Species In: DOYLE, M. P.; BEUCHAT, L. R.; MONTVILLE, T. J. Food Microbiology: fundamentals and frontiers. Washington: ASM Press, 1997.

[7] IHA, M.H.; FÁVARO, R. M. D.; OKADA, M. M.; PRADO, S. P. T. MARTINS, A. M. B.; OLIVEIRA, M. A.; FEBRONIO, L. H. P.; GARRIDO, N.S. Avaliação físico-química e higiênico-sanitária do suco de laranja não pasteurizado, engarrafado e comercializado nas cidades de Ribeirão Preto e Araraquara - SP. XVI Congresso Brasileiro de Ciência e Tecnologia de Alimentos - SBCTA, Rio de Janeiro, 1998.

[8] INSTITUTO ADOLFO LUTZ. Normas analíticas do Instituto Adolfo Lutz. 3 ed. São Paulo: IAL, 1981. V.1: Métodos químicos e físicos para análise de alimentos.

[9] KATZ, F.; GIESE, J. Science gives specialty juice a big slice of the market. Food Technology, v.52, n. 11, p. 44-48, 1998.

[10] LINTON, M.; McCLEMENTS, J. M. J.; PATTERSON, M. F. Inactivation of Escherichia coli $\mathrm{O} 157: \mathrm{H} 7$ in orange juice using a combination of high pressure and mild heat. Journal of Food Protection, v. 62, n. 3, p. 277-279, 1999.

[11] NASCIMENTO, D., do; FURLANETTO, S. M. P. Determinação quantitativa de grupos de bactérias em sucos de laranja natural. Revista de Saúde Pública, v. 15, 1981. p. 231-232, 1981.

[12] OBETA, J. A. N.; UGWUANYI, J. O. Shelf life study of some Nigerian fruit juices inoculated with ascospores of Neosartorya spp. Plant Foods for Humans Nutrition v.50, p.325-331, 1997.

[13] PAO, S.; DAVIS, C. Enhancing microbiological safety of fresh orange juice by fruit immersion in hot water and chemical 
sanitizers. Journal of Food Protection, v. 62, n. 7, p. 756-760, 1999.

[14] PARISH, M. Coliforms, Escherichia coli and Salmonella serovars associated with a citrus-processing facility implicated in a salmonellosis outbreak. Journal of Food Protection, v. 61, p. 280-284, 1998.

[15] SCHEIDEGGER, C.; PIETRZAK, J.; FREI, R. Methadone diluted with contaminated orange juice or raspberry syrup as a potential source of disseminated candidiasis in drug abusers. European Journal Clinical Microbiology Infection and Disease, v.12, p. 229-231, 1993.

[16] SPECK, M.L. (Ed.). Compendium of methods for the microbiological examination of food. 2 ed. Washington: American Public Health Association, 1976.

[17] WYATT, M. K.; PARISH, M. E.; WIDMER, W. W.; KIMBROUGH, J. Characterisation of mould orange juice. Food Microbiology, v.12, p.347-355, 1995. 